\title{
ERRATUM
}

\section{Erratum to: Distinct demographic factors influence the acceptance of vaccination against HPV}

Theodoros Agorastos - Kimon Chatzistamatiou - Menelaos Zafrakas • Vagia Siamanta - Taxiarchis Katsamagkas - Theodoros Constantinidis • Alexandros Lampropoulos • The Lysistrata study group

Published online: 29 January 2015

(c) Springer-Verlag Berlin Heidelberg 2015

\section{Erratum to: Arch Gynecol Obstet}

\section{DOI 10.1007/s00404-015-3614-4}

Unfortunately, the authors' first and family names were swapped in the official online publication and it has been corrected. The correct author names are given below.

Theodoros Agorastos - Kimon Chatzistamatiou ·

Menelaos Zafrakas · Vagia Siamanta .

Taxiarchis Katsamagkas . Theodoros Constantinidis Alexandros Lampropoulos - The Lysistrata study group

The online version of the original article can be found under doi: 10.1007/s00404-015-3614-4.

T. Agorastos $\cdot$ K. Chatzistamatiou $\cdot$ V. Siamanta .

T. Katsamagkas

4th Department of Obstetrics and Gynecology, Hippokrateio

General Hospital, Aristotle University of Thessaloniki,

Konstantinoupoleos, 49, 54642 Thessaloniki, Greece

Present Address:

K. Chatzistamatiou $(\square)$

Mousson 9, 55236 Thessaloniki, Greece

e-mail: kimon.chatzistamatiou@gmail.com

M. Zafrakas

School of Health and Medical Care, Alexander Technological

Educational Institute of Thessaloniki, Thessaloniki, Greece

T. Constantinidis

Laboratory of Hygiene, Medical School, Democritus University

of Thrace, Alexandroupolis, Greece

A. Lampropoulos

1st Department of Obstetrics and Gynecology, Papageorgiou

General Hospital, Aristotle University of Thessaloniki,

Thessaloniki, Greece 